\title{
FBG hydrogen sensor based on spiral microstructure ablated by femtosecond laser
}

\author{
Xian Zhou, Yutang Dai *, Meng Zou, Joseph Muna Karanja, Minghong Yang \\ National Engineering Laboratory for Fiber Optic Sensing Technology, Wuhan University of Technology, Wuhan, \\ 430070, China. \\ *Corresponding author: daiyt68@163.com
}

\begin{abstract}
This paper proposed and demonstrated fiber Bragg grating (FBG) hydrogen sensor by integrating optical fiber laser micro-machining and thin film technology. The spiral microstructure ablated sensor probe was sputtered with hydrogen sensitive material, palladium-silver ( $\mathrm{Pd}-\mathrm{Ag})$ composite film, in which the atomic ratio of the two metals is controlled at $\mathrm{Pd}: \mathrm{Ag}=4: 1$. Experimental work was conducted at ambient temperature and relative humidity of $25^{\circ} \mathrm{C}$ and $32 \%$ respectively. Double spiral microstructure at pitch $90 \mu \mathrm{m}$ and sputtered with $520 \mathrm{~nm} \mathrm{Pd} / \mathrm{Ag}$ film recorded hydrogen sensitivity of $51.5 \mathrm{pm} / \% \mathrm{H}$ responding to hydrogen gas in air. This translated to approximately 7.5 times higher than the unprocessed standard FBG. The sensor's hydrogen response is reversible, has improved response time and could have potential for hydrogen gas monitoring.
\end{abstract}

Key words: Pd/Ag alloy film; femtosecond laser; hydrogen sensor; spiral microstructure; fiber Bragg grating

\section{Introduction}

Compared to traditional sensors based on electrical features, optical fiber sensors have distinct advantages such as intrinsically safe, high sensitivity, strong stability, small volume and lightweight. Since Butler[1] at Sandia national laboratories made the world's first optical fiber hydrogen sensor, many kinds of optical fiber hydrogen sensors have been developed, such as micro-mirror sensor[2-3], interference sensor[4-5], evanescent sensor[6], surface plasmon resonance sensor[7-8] and fiber Bragg grating(FBG) sensor[9-13]. Due to obvious advantages in wavelength division multiplex (WDM), self-reference and anti-interference, FBG sensor is more suitable for monitoring hydrogen leak for distribution measurement.

At present, there are many types of hydrogen sensing materials, one type is metallic oxide based on mechanism of resistivity change or gasochromic effect, such as Perovskite-Type Oxide, Indium-tin oxide, $\mathrm{WO}_{3}$ and so on, while another type is metal or metal alloy such as Pd or Pd alloys. Chemical interaction between $\mathrm{WO}_{3}$ and hydrogen is not strong enough and requires initial exothermic reaction. Tungsten trioxide also chemically interact with other hydrogen based compounds specimen, such as $\mathrm{H}_{2} \mathrm{~S}$, $\mathrm{C}_{2} \mathrm{H}_{2}$ etc., and therefore does not show selective sensitivity to hydrogen. On the other hand, compared to metallic oxide, hydrogen sensors based on Pd or Pd alloys have simple film structure, and it's also easier to sputter Pd or Pd alloys thin film. These sensors can be used to detect hydrogen concentration when they are exposed to hydrogen gas, since, thin films can produce physical and chemical reaction with hydrogen, leading to mechanical stress and optical parameters alternation of the fiber. Despite the superior sensitivity of pure Pd based hydrogen sensors, several cycles of absorption and desorption of hydrogen causes Pd layer to peel off from the cladding, crack and form blisters due to $\alpha-\beta$ phase transition[10, 12, 14]. In addition, sensors have longer response time using pure Pd film as sensing material. The sensor based on Pd alloy such as Pd-Ni[15-16], Pd-Au[6, 17], Pd-Ag[13, 18-20], can suppress $\alpha-\beta$ transition to some extent and improve hydrogen permeating capacity. Among these Pd alloys, Pd-Ag composite film has attracted wide attention in film technology because of its better 
hydrogen selectivity and permeability than above mentioned alloy films and provides a longer operational lifetime[21]. If Pd-Ag film is coated on a FBG fiber, it experiences expansion as absorbs hydrogen gas. Meanwhile, the FBG underneath experiences strain that results to FBG wavelength shift, therefore, the hydrogen concentration can be detected. Generally, the tensile stress caused by Pd-Ag film swelling is so weak that the sensitivity of FBG sensor is marginal, therefore, how to improve the sensitivity would be a key issue for hydrogen sensor based on FBG. Some researchers have used chemical etching method to decrease the diameter of fiber cladding on which Pd or Pd alloy thin film was coated[10, 22-23], others considered decreasing the fiber cross-section by taper technology and side-polished method[20, 24-25], and the sensitivity of these sensors were improved to some extent. However, it is difficult to operate too thin fiber since the fiber sensor becomes very fragile.

In order to improve sensitivity and response time of the FBG fiber hydrogen sensor, we made a novel FBG fiber hydrogen sensor by etching spiral microstructure on the FBG fiber cladding and sputtering Pd-Ag film into the microgroove. Femtosecond (fs) laser is employed to make spiral microstructure due to its excellent processing property for transparent materials[26]. Hydrogen sensor probes with spiral microstructure were successfully developed, and the results show that the sensitivity was greatly improved.

\section{Principle}

Compared to non-microstructured FBG, the sensitivity of etched FBG sensor can be improved significantly due to its increased surface area and better stretchability. As shown in Fig.1, the main parameters for sensor probe are: diameter of cladding- $D$, microgroove depth- $d$, width- $w$, length of the slop- $c$, spiral microgroove pitch- $p$, total spiral microgroove probe length-l. The increased area for double spiral microstructure fiber with length $l$ is expressed by

$$
\begin{aligned}
& s_{i}=\frac{2\left(2 s_{c}-s_{w}\right) l}{p} \\
& s_{w}=w * l_{w}=w * \sqrt{\pi^{2} D^{2}+p^{2}} \\
& s_{c}=c * l_{c} \approx c \sqrt{\pi^{2}(D-d)^{2}+p^{2}}
\end{aligned}
$$

Where, helical surface $s_{w}$ is generated by the straight line with length of $w$ rotating a pitch length around the axis, and the slope of microgroove $s_{c}$ is generated by the oblique line $c$ as well. $l_{w}$ is the helix length for one pitch, $l_{c}$ is the helix line length for one pitch generated by the midpoint $\mathrm{m}$ of oblique line $c$. Double spiral micrstructured FBG have double increased area than that of single spiral.

Pd-Ag composite film expansion leads to strain produced inside of the FBG fiber which approximate equal to uniform axial strain, sensitivity of wavelength shift due to uniform axial strain can be expressed as

$$
\frac{\Delta \lambda_{B}}{\lambda_{B}}=\left(1-p_{e}\right) \varepsilon
$$

Where $p_{e}$ is the valid elastic-optic constants, $\varepsilon$ is the axial strain. Assuming that the strain of the palladium alloy film is $\varepsilon_{p d}$ generated by film expansion when it absorbs hydrogen, subsequent the resulting in the fiber tension is

$$
F=\varepsilon_{p d} Y_{p d} A_{p d}
$$

Where $Y_{p d}$ is Young modulus of palladium, $A_{p d}$ is the palladium film cross section area. The strain in the fiber can be described as 


$$
\varepsilon_{f}=\frac{F}{Y_{p d} A_{p d}+Y_{f} A_{f}} \approx \frac{F}{Y_{f} A_{f}}=\frac{F}{Y_{f} \pi(D-2 d)^{2}}
$$

Where $Y_{f}$ and $A_{f}$ is the optical fiber Young modulus and cross section area respectively, assuming $A_{p d}=0\left(A_{f} \gg A_{p d}\right)$. The wavelength shift of Pd-Ag coated FBG can be obtained by combining Eq.(4), Eq.(5) and Eq.(6):

$$
\begin{gathered}
\Delta \lambda_{B}=\frac{\left(1-p_{e}\right) F \lambda_{B}}{Y_{f} A_{f}}=\frac{\left(1-p_{e}\right) \varepsilon Y_{p d} A_{p d} \lambda_{B}}{Y_{f} \pi(D-2 d)^{2}} \\
\Delta \lambda_{s}=\frac{\left(1-p_{e}\right) F_{s} \lambda_{B}}{Y_{f} A_{f}}=\frac{\left(1-p_{e}\right) \varepsilon_{s} Y_{p d} A_{p d} \lambda_{B}}{Y_{f} \pi D^{2}}
\end{gathered}
$$

The sensitivity promotion ratio $(\mathrm{R})$ relative to the standard FBG could be calculated as

$$
R=\frac{\Delta \lambda_{B}}{\Delta \lambda_{s}}=\frac{\varepsilon_{p d} D^{2}}{\varepsilon_{s}(D-2 d)^{2}}
$$

Where $\Delta \lambda_{s}, F_{s}$ and $\varepsilon_{s}$ is wavelength shift associated with standard FBG, axial force and strain of the palladium alloy film from the sensitive film affected by the measurand on the standard FBG respectively. The coated surface area of the micro-structured sensor probe is thus greater than that of standard FBG. As a result, $\varepsilon_{p d}>\varepsilon_{s}$, and conclusively $R \gg 1$. According to equation (9), the deeper the microgroove, the more sensitive the sensors. Correspondingly, the sensitive of double spiral microstructured probe is higher than that of single spiral. If considering the stress inside the fiber induced by the laser, the sensitive is even higher.

\section{Experiment}

\section{1 spiral microstructure preparation}

The FBG $10 \mathrm{~mm}$ long was written into the core of standard single mode fiber by first removing polyimide layer, then irradiating the fiber using a $\mathrm{KrF}$ excimer $(248 \mathrm{~nm})$ laser by phase mask technique. Spiral microstructure was fabricated on the FBG cladding as $10 \mathrm{~mm}$ long by a commercial amplified Ti: sapphire laser micromachining system made by Cyber Laser, Japan The micromachining system consists of IFRIT laser (wavelength at $780 \mathrm{~nm}$, pulse duration of $180 \mathrm{fs}$, repetition rate of $1 \mathrm{kHz}$ ), three-dimensional table, optical system and CCD monitoring system. The maximum pulse energy was $1.08 \mathrm{~mJ}$. The energy density at the fiber position was controlled from $1 \%$ to $100 \%$ by an optical attenuator and a diaphragm. The specimen was mounted on a high-precision three-axis computer driven motorized stage. In addition, a specifically designed rotating jig is used to hold and drive the optical fiber. The laser beam was focused on the fiber surface using an objective lens (Sigama, Koki, Japan) with a focal length of $60 \mathrm{~mm}$, the entire process of the laser ablation was monitored real-time by a CCD camera. The schematic of whole laser system is shown in Fig.2. When the fiber was ablated, the fiber was rotated around its axis at a constant rotational speed $\omega$ while stage has uniform speed $v$ along the fiber axis, therefore, a 3D-sprial microstructure was made with a constant pitch that can be expressed:

$$
p=\frac{v}{\omega}
$$

Especially, the laser spot was focused at $25 \mu \mathrm{m}$ offset from the center of the fiber core to avoid the deformed spectrum. The laser energy fluence and the spot motion speed determine the depth of the spiral micro-grooves. The laser pulse power was set from 0.25 to $0.35 \mathrm{~mW}$ at step $0.05 \mathrm{~mW}$, repetition rate $1000 \mathrm{~Hz}$, the rotation speed $\omega$ was set 12 r/min and the stage feed rate was $1.44,1.08,0.72 \mathrm{~mm} / \mathrm{min}$, respectively. Finally, FBG specimen were prepared with spiral pitch at 120 , 90 , $60 \mu \mathrm{m}$ 
respectively. All samples parameters are shown in Table 1.

Table 1. Parameters of tested samples

\begin{tabular}{llll}
\hline Specimen & $\begin{array}{l}\text { Power } \\
(m w)\end{array}$ & $\begin{array}{l}\text { Pitch } \\
(\mu m)\end{array}$ & $\begin{array}{l}\text { Depth } \\
(\mu m)\end{array}$ \\
\hline s-1 & 30 & 60 & 19 \\
s-2 & 30 & 90 & 19 \\
s-3 & 30 & 120 & 19 \\
ss-1 & 35 & 90 & 21 \\
ss-2 & 30 & 90 & 19 \\
ss-3 & 25 & 90 & 16.5 \\
ss-4 & 25 & 60 & 16.5 \\
ss-5 & 25 & 120 & 16.5 \\
Standard & 0 & 0 & 0 \\
FBG & & & \\
\hline
\end{tabular}

\subsection{Pd-Ag film sputtered}

The micro-structured fiber is sputtered with hydrogen sensitive film after spiral microstructures fabrication. Before sputtering, samples were subjected into the ultrasonic cleaner with solution HF in 0.5 minutes for cleaning the debris on the surface of the cladding. A BESTECH sputtering system equipped with DC and RF sputtering sources was used to prepare thin films, the laser ablated fibers were placed under the substrate-target of $15 \mathrm{~cm}$. Meanwhile, two $10 \times 10 \mathrm{~mm}$ Si pieces were used to evaluate the film depth and analyze the content of alloy element. Pd-Ag film thick $500 \mathrm{~nm}$ were coated. In order to enhance the adhesion of Pd-Ag films on the FBG fiber, $20 \mathrm{~nm} \mathrm{Ni}$ was first deposited on surface of FBG fiber by RF sputtering process to ensure the uniform of deposited films, all samples were turned $180^{\circ}$ after depositing $250 \mathrm{~nm}$ thick Pd-Ag thin film. Under $0.5 \mathrm{~Pa}$ sputtering pressure of $\mathrm{Ar}$, the deposition power for $\mathrm{Pd}$ and $\mathrm{Ag}$ targets were start 125 and $80 \mathrm{~W}$ respectively, which corresponds to deposition rate of 1.8 and $0.5 \AA$ /s respectively. After sputtering process, the atomic ratio of Pd and $\mathrm{Ag}$ is about 80:20 by using Energy Dispersive Spectrometer (EDS) for quantitive elemental analysis. The SEM images of single spiral and double spiral microstructured probe is shown in Fig.3.

\section{3 hydrogen gas testing system}

The schematic diagram of hydrogen test system is shown in Fig.4. The characterization was performed at constant room temperature $\left(25^{\circ} \mathrm{C}\right)$ and the relative humidity of the atmosphere is about $32 \%$. Hydrogen concentration was calibrated by using commercial electrochemical hydrogen meter (RBT-6000 -ZLG/A; Ruian Company, China), fitted with alarm to respond to $\mathrm{H}_{2}$ concentration beyond $4 \%$ in the chamber. A hydrogen concentration meter based on chemical electrics principles was connected to the chamber for calibration. The sensors in the chamber were connected to a FBG demodulator based on CCD demodulation principle, which has high precision to detect $1 \mathrm{pm}$ wavelength shift. The output port of the demodulator was connected to a computer to collect and analyze the measured data. The thermal cross-sensitivity of the FBG sensor must be eliminated if the FBG hydrogen sensor is applied in practice. This can be achieved by connecting a reference FBG (standard FBG) in series with micro-structured FBG to compensate the ambient temperature change, thus, the effect of temperature can be overcome. However, in our laboratory test, we maintained constant room temperature $\left(25^{\circ} \mathrm{C}\right)$, thus, thermal cross-sensitivity issue was easily solved. 


\section{Results and discussion}

Taking s-2 as an example, Fig.5 shows the spectrum under different situations using high precision spectrometer. It can be observed that the reflective spectrum is permanent red shift about $0.5 \mathrm{~nm}$ after laser ablating and there is no evident deformation and optical power loss. Meanwhile, there seems no difference between spectrum of FBG before and after sputtering film.

Fig.6 (a) shows the result of element analysis the pattern of Pd-Ag composite film deposited on the surface of etching FBG by EDS using the field emission scanning electron microscope (Zeiss Ultra plus, Germany). The EDS result shows the atom ratio of $\mathrm{Pd} / \mathrm{Ag}$ is about 80.1/19.9, which is basically same as elements ratio designed at 4/1. Fig.6 (b) displays the FE-SEM picture of $\mathrm{Pd} / \mathrm{Ag}$ film after sputtering. The white particles are Ag atoms and the gray particles are Pd atoms, it can be seen that the surface is smooth, uniform and highly dense.

Fig.7 displays the ss-1, ss-2 hydrogen sensing performance of $500 \mathrm{~nm}$ depth $\mathrm{Pd} / \mathrm{Ag}$ films examined at room temperature $25^{\circ} \mathrm{C}$ using air as carrier gases for different hydrogen concentrations. Fig.7 (a) illustrates good repeatability of ss-1 in hydrogen response cycles. The central wavelength have a 1-2 pm higher compared to origin central wavelength after hydrogen gas out for s-1 sensor, the reason is may due to irreversible structure deformation in Pd film for phase transition, the deformed crystal structure could not recover after $\mathrm{H}$ atoms desorbed from the hydrogenated Pd. Addition of $\mathrm{Ag}$ atoms to the Pd film can suppress the phase transition, however, it cannot completely eliminate it. However, annealing can improve performance of the crystal structure such as grain growth and stress relaxation[2], therefore, by adopting the method of annealing can indirectly ease the original central wavelength shift phenomenon.

The wavelength shift of ss- 1 sensor toward $4 \% \mathrm{H}_{2}$ is $210 \mathrm{pm}$. For ss- 1 and ss-2 the response time is $160-180 \mathrm{~s}$ at $4 \% \mathrm{H}_{2}$, while recovery time is $60-80 \mathrm{~s}$. The response time is faster than the reported results about 280-300 s[18]. The response time and recovery time are defined as that required for $100 \%$ of corresponding signal change for absorption and desorption of hydrogen. Response time is influenced by many factors such as film thickness, hydrogen pressure, temperature, humidity and so on. The faster response time may be explained as follows, firstly, $\mathrm{Ag}$ atoms content can influence the permeation of the composite $\mathrm{Pd} / \mathrm{Ag}$ film to hydrogen, ref.[21] reported the maximum hydrogen diffusion rate with silver content is around 20-23 percent of the composite $\mathrm{Pd} / \mathrm{Ag}$ film at $350^{\circ} \mathrm{C}$, it's simply infers that the hydrogen diffuse rate is may faster at $20 \% \mathrm{Ag}$ atoms content compared to $25 \% \mathrm{Ag}$ content at room temperature, and this should be verified and investigated in future. Secondly, the humidity can influence the $\mathrm{H}$ atoms rate of diffusion. Increase in relative humidity, lowers the $\mathrm{H}$ atoms diffusion rate and vice versa[3, 27]. Thus, our current work performed at lower humidity has higher $\mathrm{H}$ atoms diffusion rate compared with reported sensor characterized at $61.7 \%$ relative humidity[18]. If relative humidity increases, the sensitivity of sensor will be decreased. We tested the sensor at different relative humidity of 32 and $65 \%$ and realized maximum wavelength shift of 133 and 84 pm respectively for the sample ss-2. This result was in agreement that high relative humidity causes hydrogen insensitivity as reported by $[18,27,29]$. In addition, the proposed sensor has shorter response and recovery time. Generally, the recovery time can be greatly enhanced in an oxygen atmosphere and is shorter compared to the response time. Since the present work uses air as carrier gas, the oxygen in the atmosphere can significantly enhance desorption rate by the formation of $\mathrm{OH}^{-}$and $\mathrm{H}_{2} \mathrm{O}$ molecules induced by the reaction between the surface of $\mathrm{Pd} / \mathrm{Ag}$ film and oxygen[28].

Fig.7 (b) demonstrates hydrogen response of ss-2 under different hydrogen concentrations beginning from ambient air condition, the wavelength shift are $25,70,100,133 \mathrm{pm}$ toward $1 \%, 2 \%, 3 \%, 4 \%$ of hydrogen respectively at $25^{\circ} \mathrm{C}$. The fluctuation of the wavelength shift of FBG is about $3 \mathrm{pm}$. The sensor response fall back to baseline when hydrogen gas is out of the gas chamber. This phenomenon demonstrates the sensor's reversibility for the 1-4\% hydrogen concentrations. 
As illustrated in Fig.8 (a), the central wavelength shift of FBG samples fabricated with different power at different hydrogen concentrations. The FBG hydrogen sensors show a linear response between $1 \%-4 \% \mathrm{H}_{2}$. The sensitivity of sample ss-1, ss-2, ss-3 is $52.5,33.3,25.5 \mathrm{pm} / \% \mathrm{H}$ respectively, while the standard FBG sensor is $7 \mathrm{pm} / \% \mathrm{H}$. Apparently, the double spiral microstructured FBG sensor significantly improves the sensitivity of hydrogen sensor. This can be explained from two main reasons. Firstly, the section of fiber cladding is decreased due to laser ablation resulting to increasing the retractility of fiber. Hence, the double spiral microstructured FBG hydrogen sensor is easy to stretch compared the standard FBG hydrogen sensor under the same tension. Secondly, due laser machining Gauss energy distribution, V-shaped microgroove formed has increasing fiber cladding surface area according to equation (1). As a result, more axial stress is formed due to more Pd/Ag particles coated on the cladding surface of the fiber. Through integration of increased $\mathrm{Pd} / \mathrm{Ag}$ films coated surface area and improved local fiber retractility, the performance of double spiral microstructured FBG hydrogen sensors are improved greatly than that of standard FBG hydrogen sensor. It can be also observed that the hydrogen sensitivity of ss-1 is the highest, followed by ss-2 and ss-3. It demonstrates that higher laser energy can improve the sensitivity of the hydrogen sensors. The main reason is that the higher laser energy makes deeper microgrooves, thus improving the flexibility of the fiber.

Fig.8 (b), (c) displays the relationship between the wavelength shift and pitch of spiral microgroove. Both double and single spiral sensor with pitch $60 \mu \mathrm{m}$ have maximum sensitivity to hydrogen, followed by the pitch of 120 and $90 \mu \mathrm{m}$. It is due to the combined effect of flexibility of FBG sensor and axial component of strain. When the pitch is smaller, the flexibility of fiber will be larger, but the axial component of strain becomes smaller along the fiber.

It is obvious that the sensitivity of double spiral sensor is higher than that of single spiral sensor under the same processing parameters shown in Fig.8 (d), the sensitivity of sample ss-2 is about 1.5 times that of sample s-2. The reason is that double spiral microstructure FBG sensor has more surface area and thus more flexibility compared to the single spiral FBG sensor. Generally speaking, double spiral type sensors have higher sensitivity which show great prospect in hydrogen sensing field.

Additionally, Fig.8 (a), (b), (c) also shows that, the maximum wavelength shift of the double spiral is 38,32 and 30 pm under the concentration of $1 \%$, corresponding to samples ss- 4 , ss-2 and ss-3, respectively. The maximum wavelength shift detected by the single spiral micro-structured sensors are 27, 18 and 12 pm, corresponding to samples s-1, s-3 and s- 2 respectively. For sample ss-4, the wavelength shift is 4,10 and $38 \mathrm{pm}$ toward $0.3 \%, 0.5 \%$ and $1 \% \mathrm{H}_{2}$, respectively. The results of two cycle's response towards $0.3 \%$ are shown in Fig.9. Due to the limited resolution of demodulator, the response waveform seems non-uniform, however, the trend of wavelength change is obviously increased or decreased when the hydrogen gas is absorbed or desorbed. The response time is about $30 \mathrm{~s}$ and contribute to the lower hydrogen gas pressure. Therefore, the hydrogen atom can't permeate deeper into the $\mathrm{Pd} / \mathrm{Ag}$ alloy film. According to the process of testing, when the hydrogen gas concentration is increasing to $0.1 \%$, the wavelength shift is about $1-2 \mathrm{pm}$, which is impossible to determine whether the wavelength change is caused by the hydrogen gas concentration due to some interference signal of the demodulator. When the hydrogen concentration is up to $0.2 \%$, we can observe the upward tendency of the wavelength shift signal. Therefore, the resolution of the hydrogen sensor is about $200 \mathrm{ppm}$ and the lowest hydrogen concentration can be measured at $0.2 \%$.

\section{Conclusion}

Spiral microstructured FBG hydrogen sensor based on $520 \mathrm{~nm} \mathrm{Pd/Ag} \mathrm{composite} \mathrm{film} \mathrm{have} \mathrm{been} \mathrm{proposed} \mathrm{and} \mathrm{demonstrated.}$ The sensitivity of hydrogen sensor was greatly improved using femtosecond laser ablated microgroove in the cladding of the fiber. The Pd/Ag composite film with atomic ratio 4:1 has faster response time. Two types of microstructure have been tested in the hydrogen gas chamber. Double Spiral probe have strongest response to hydrogen gas, followed by single spiral type sensor, standard FBG type, and all the samples have good repeatability and faster response rate during the hydrogen response. The resolution of the hydrogen sensor is about $200 \mathrm{ppm}$ and the lowest hydrogen concentration can be measured at $0.2 \%$. Laser 
ablation of the spiral microgroove is very efficient, which is a quite promising for hydrogen sensor application.

\section{Acknowledgments}

This work is financially supported by the Project of National Science Foundation of China, NSFC (Number: 61475121 and No.61290311).

\section{References:}

[1] M.A. Butler, Optical fiber hydrogen sensor, Appl. Phys. Lett. 45 (1984) 1007.

[2] H. Song, Y. Chen, G. Zhang, Y. Liu, P. Huang, H. Zhao, M. Yang, J. Dai and Z. Li, Optical fiber hydrogen sensor based on an annealing-stimulated Pd - Y thin film, Sens. Actuators B 216 (2015) 11-16.

[3] M. Slaman, Optimization of Mg-based fiber optic hydrogen detectors by alloying the catalyst, Int. J. Hydrogen Energ. 33 (2008) 1084-1089.

[4] M. Wang, M. Yang, J. Cheng, J. Dai, M. Yang and D.N. Wang, Femtosecond laser fabricated micro Mach-Zehnder interferometer with Pd film as sensing materials for hydrogen sensing, Opt. Lett. 37 (2012) 1940-1942.

[5] Y. Wang, M. Yang, G. Zhang, J. Dai, Y. Zhang, Z. Zhuang and W. Hu, Fiber Optic Hydrogen Sensor Based on Fabry Perot Interferometer Coated With Sol-Gel Pt/WO 3 Coating, J. Lightwave Technol. 33 (2015) 2530-2534.

[6] D. Luna-Moreno, D. Monzón-Hernández, J. Villatoro and G. Badenes, Optical fiber hydrogen sensor based on core diameter mismatch and annealed Pd - Au thin films, Sens. Actuators B 125 (2007) 66-71.

[7] R. Tabassum and B.D. Gupta, Surface plasmon resonance-based fiber-optic hydrogen gas sensor utilizing palladium supported zinc oxide multilayers and their nanocomposite, Appl. Opt. 54 (2015) 1032-1040.

[8] A. Hosoki, M. Nishiyama, H. Igawa, A. Seki and K. Watanabe, A hydrogen curing effect on surface plasmon resonance fiber optic hydrogen sensors using an annealed $\mathrm{Au} / \mathrm{Ta}_{2} \mathrm{O}_{5} / \mathrm{Pd}$ multi-layers film, Opt. Express 22 (2014) 15563-18556.

[9] S. Masuzawa, S. Okazaki, Y. Maru and T. Mizutani, Catalyst-type-an optical fiber sensor for hydrogen leakage based on fiber Bragg gratings, Sens. Actuators B 190 (2015) 151-157.

[10] B. Sutapun, M. Tabib-Azar and A. Kazemi, Pd-coated elastooptic fiber optic Bragg grating sensors for multiplexed hydrogen sensing, Sens. Actuators B 217 (1999) 27-34.

[11] J. Dai, M. Yang, Z. Yang, Z. Li, Y. Wang, G. Wang, Y. Zhang and Z. Zhuang, Performance of fiber Bragg grating hydrogen sensor coated with Pt-loaded WO3 coating, Sens. Actuators B 60 (2014) 657-663.

[12] G. Ma, C. Li, Y. Luo, R. Mu and L. Wang, High sensitive and reliable fiber Bragg grating hydrogen sensor for fault detection of power transformer, Sens. Actuators B 169 (2012) 195-198.

[13] R.R.J. Maier, B.J.S. Jones, J.S. Barton, S. McCulloch, T. Allsop, J.D.C. Jones and I. Bennion, Fibre optics in palladium-based hydrogen sensing, J. Opt. A-Pure Appl. Opt. 9 (2007) S45-S59.

[14] M. Wang and Y. Feng, Palladium - silver thin film for hydrogen sensing, Sens. Actuators B 23 (2007) 101-106.

[15] Y.J. Ou, W.W. Si, G. Yu, L.L. Tang, J. Zhang and Q.Z. Dong, Nanostructures of Pd - Ni alloy deposited on carbon fibers for sensing hydrogen, J. Alloy Compd. 569 (2013) 130-135.

[16] J. Dai, M. Yang, X. Yu, K. Cao and J. Liao, Greatly etched fiber Bragg grating hydrogen sensor with Pd/Ni composite film as sensing material, Sens. Actuators B 174 (2012) 253-257.

[17] D. Luna-Moreno and D. Monzón-Hernández, Effect of the Pd - Au thin film thickness uniformity on the performance of an optical fiber hydrogen sensor, Appl. Surf. Sci. 253 (2007) 8615-8619.

[18] J. Dai, M. Yang, X. Yu and H. Lu, Optical hydrogen sensor based on etched fiber Bragg grating sputtered with Pd/Ag composite film, Opt. Fiber Technol. 19 (2013) 26-30.

[19] G.M. Ma, J. Jiang, C.R. Li, H.T. Song, Y.T. Luo and H.B. Wang, Pd/Ag coated fiber Bragg grating sensor for hydrogen monitoring in power transformers, Rev. Sci. Instrum. 86 (2015) 45003. 
[20] J. Jiang, G. Ma, C. Li, H. Song, Y. Luo and H. Wang, Highly Sensitive Dissolved Hydrogen Sensor Based on Side-Polished Fiber Bragg Grating, IEEE Photonic. Tech. Lett. 27 (2015) 1453-1456.

[21] A.G. Knapton, Palladium Alloys for Hydrogen Diffusion Membranes, Platin. Met. Rev. 04 (1977) 21-22.

[22] M. Aleixandre, P. Corredera, M.L. Hernanz, I. Sayago, M.C. Horrillo and J. Gutierrez-Monreal, Study of a palladium coated Bragg grating sensor to detect and measure low hydrogen concentrations, 2007 Spanish Conference on Electron Devices, Proceedings (2007) 223-225.

[23] J. Dai, M. Yang, X. Yu, K. Cao and J. Liao, Greatly etched fiber Bragg grating hydrogen sensor with Pd/Ni composite film as sensing material, Sens. Actuators B 174 (2012) 253-257.

[24] S. Silva, L. Coelho, J.M. Almeida, O. Frazao, J.L. Santos, F.X. Malcata, M. Becker, M. Rothhardt and H. Bartelt, $\mathrm{H}_{2}$ Sensing Based on a Pd-Coated Tapered-FBG Fabricated by DUV Femtosecond Laser Technique, IEEE Photonic. Tech. Lett. 25 (2013) 401-403.

[25] J. Dai, M. Yang, Y. Chen, K. Cao, H. Liao and P. Zhang, Side-polished fiber Bragg grating hydrogen sensor with WO3-Pd composite film as sensing materials, Opt. Express 19 (2011) 6141-6148.

[26] R.R. Mazur, E. Mazur, Femtosecond laser micromachining in transparent materials, Nat. Photonics 2 (2008) 219-225.

[27] Z. Zhao, M. Knight, S. Kumar, E.T. Eisenbraun and M.A. Carpenter, Humidity effects on Pd/Au-based all-optical hydrogen sensors, Sens. Actuators B 129 (2008) 726-733.

[28] L.G. Petersson, H.M. Dannetun, I. Lundström, Hydrogen Detection during Catalytic Surface Reactions: Evidence for Activated Lateral Hydrogen Mobility in the Water-Forming Reaction on Pd, Phys. Rev. Lett. 52 (1984) .

[29] L. Coelho, J. M. M. M. de Almeida, J. L. Santos and D. Viegas, Fiber optic hydrogen sensor based on an etched Bragg grating coated with palladium, App. Opt. 54 (2015) 10342-10348. 


\section{List of Figures:}

Fig.1. Schematic diagram of single spiral and double spiral microstructure.

Fig.2. The sketch of laser system.

Fig.3. SEM of spiral microstructured FBG.

Fig.4. Schematic of hydrogen gas test system.

Fig.5. Spectrum of s-2 under different situations.

Fig.6. EDAX pattern of Pd-Ag composite films -(a) elemental composition of the Pd/Ag alloy film; (b) the SEM picture of the composite thin film.

Fig.7. Corresponding sensing responses for Pd/Ag etched FBG sensor at different hydrogen concentration. -(a) ss-1 sample; (b) ss-2 sample.

Fig.8. performance of sensors -(a) with different power; (b) with different pitch (double spiral); (c) with different pitch (single spiral); (d) single and double spiral sensor with same parameters.

Fig.9. Cycle's response to $0.3 \%$ hydrogen concentration. 


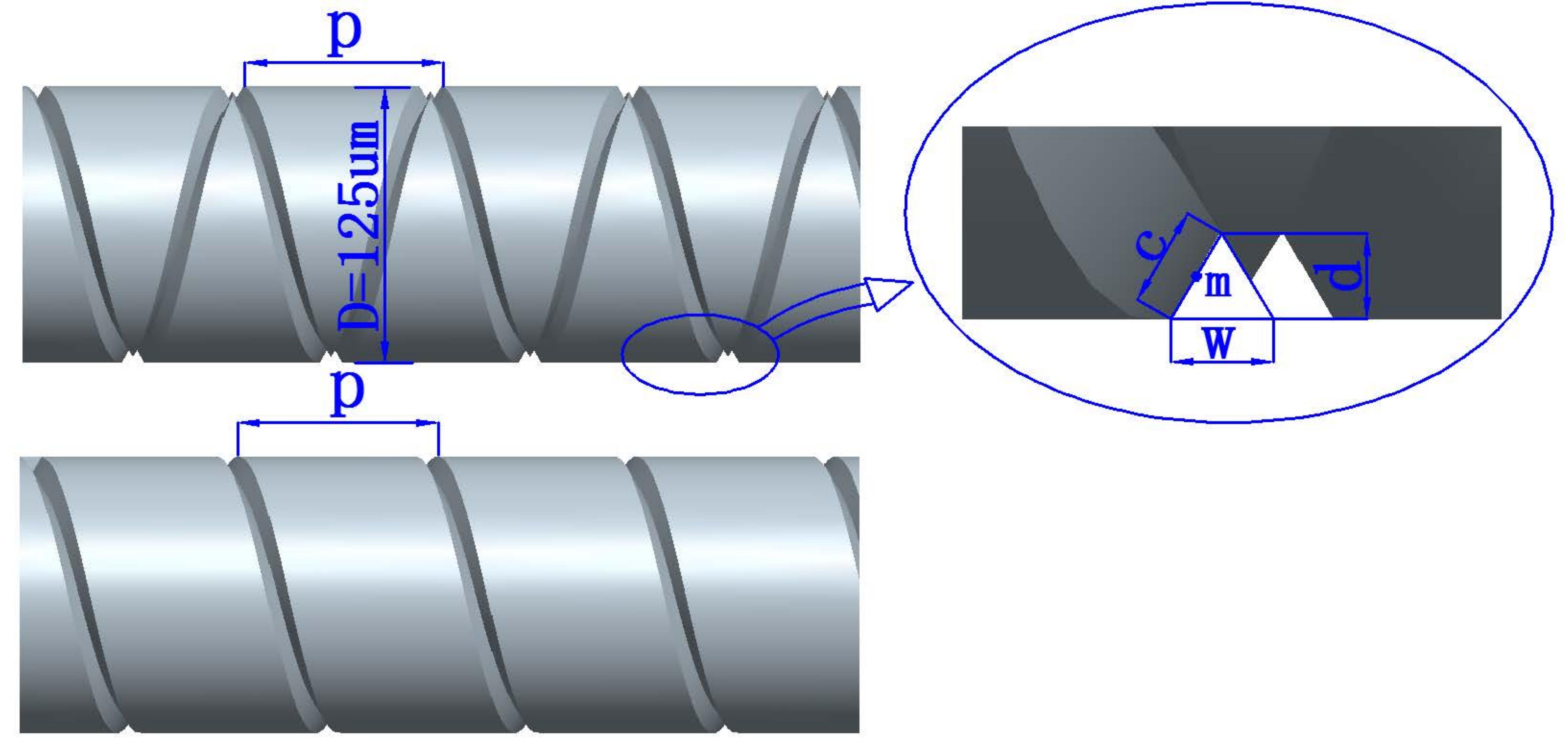



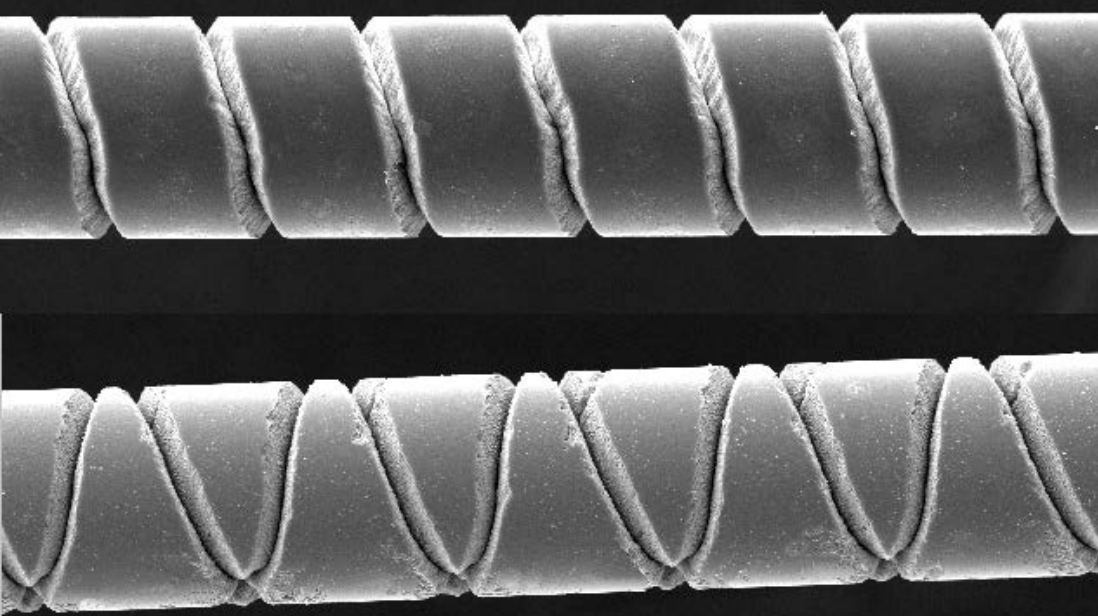

Z5kU

XZGG 1 GG

JSM-5S 1 GLU 


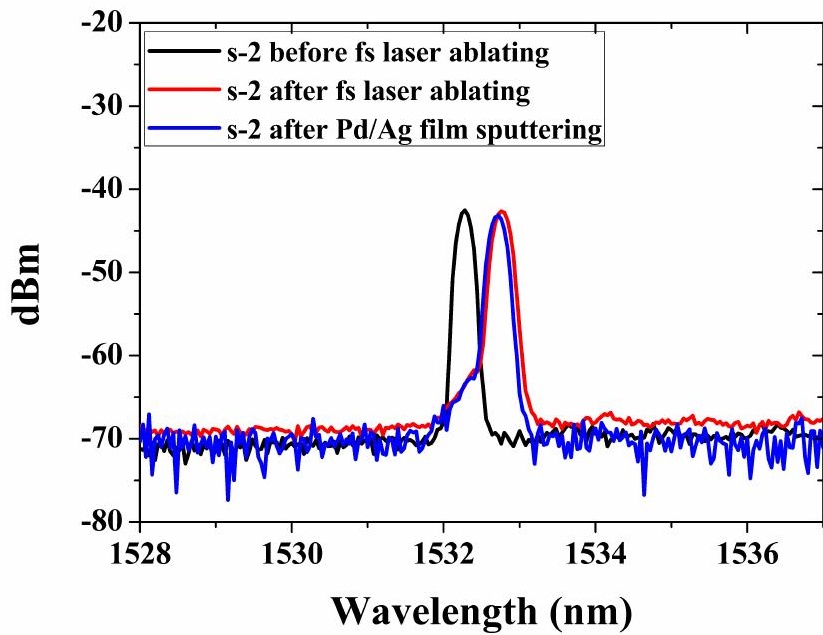




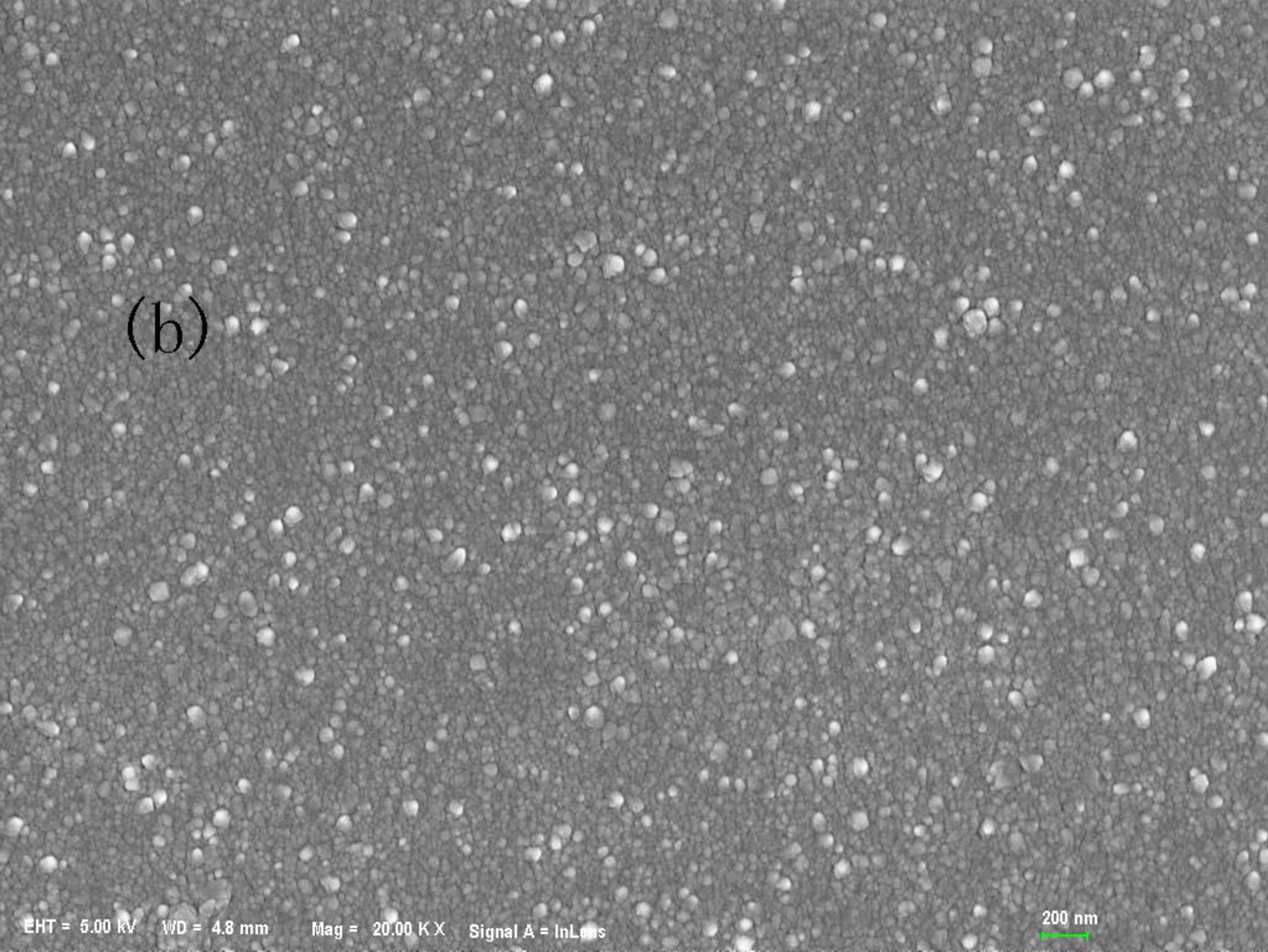




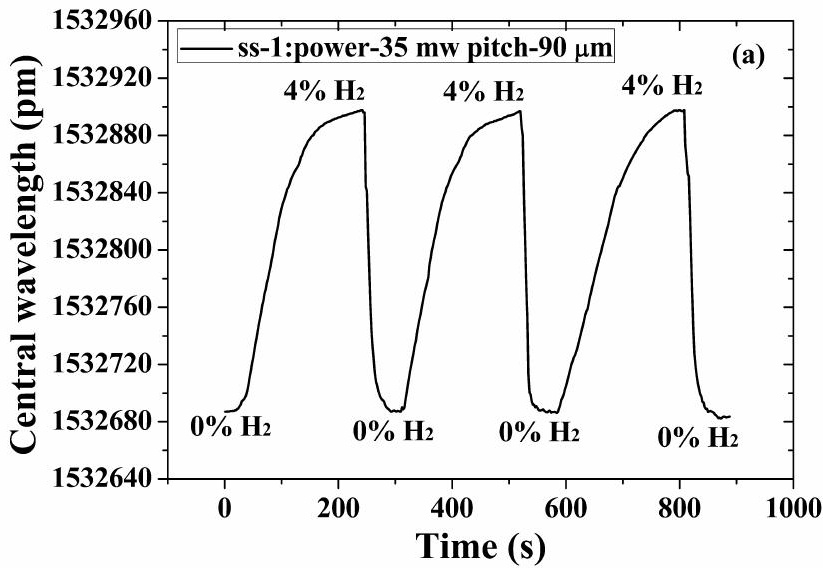




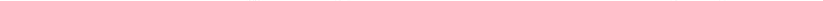




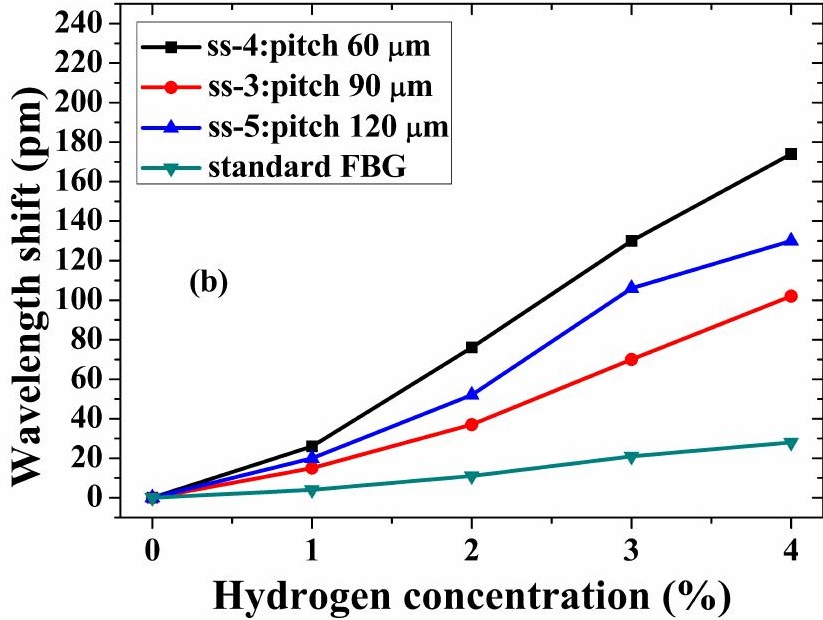




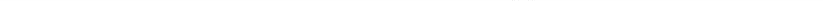


Xian Zhou was born in Jiangxi, China, in 1982. He received his Master degree in Aerospace Manufacturing Engineering from Nanchang Hangkong University. He is currently pursuing $\mathrm{PhD}$. in Wuhan University of Technology. His current research interest is in the field of optical fiber sensors and femtosecond laser processing.

Yutang Dai is currently a Professor at National Engineering Laboratory of Fiber Optical Sensing technology, Wuhan University of Technology, China. He received the Ph.D. degree from the Nippon Institute of Technology, Japan, in 2001. Then, he studied ultra-precision machining technique for 4 years in the Institute of Physical and Chemical Research, Japan. His current interests include ultra-short and ultra-fast laser micromachining of optoelectronic materials, and the development of new-type fiber optical sensors. He is author of over 30 journal publications.

Meng Zou received his first bachelor's degree in mechanical engineering from Wuhan University of Technology. He is now a graduate student with research interest in femtosecond laser processing.

J.M Karanja received his Msc in Electronics and Instrumentation from Kenyatta University (Kenya) in 2009. He is currently pursuing $\mathrm{PhD}$ in Wuhan University of Technology. His research interest concentrates on Optical Fiber Sensors.

Minghong Yang received his Ph.D. in physics from Huazhong University of Science and Technology, China, in 2003. From 2003 to 2005, he worked as a postdoctoral fellow at the Fraunhofer Institute for Applied Optics and Fine Mechanics, Jena, Germany. From 2006 to 2008, he worked as a research fellow at Berlin University of Technology, where he dealt with thin film sensors. He is now a research scientist at the National Engineering Laboratory for Optical Fiber Sensors at Wuhan University of Technology, China. His research interest is thin film optical fiber sensors. 